\section{AB0649 B CELL DEPLETION THERAPY IN VERY EARLY SYSTEMIC SCLEROSIS. A POTENTIAL WINDOW OF OPPORTUNITY?}

Dimitrios Daoussis ${ }^{1}$, Pantelis Kraniotis ${ }^{2}$, loannis Antonopoulos ${ }^{3}$ Konstantinos Melissaropoulos ${ }^{3}$, Stamatis-Nick Liossis ${ }^{1} .{ }^{1}$ Patras University Hospital, University of Patras Medical School, Rheumatology, Patras, Greece; ${ }^{2}$ Patras University Hospital, Radiology, Patras, Greece; ${ }^{3}$ Patras University Hospital, Rheumatology, Patras, Greece

Background: Our group, among others, has shown that B cell depletion therapy may improve skin thickening and interstitial lung disease (ILD) related to systemic sclerosis (SSc). All studies assessing the clinical efficacy of B cell depletion therapy in SSc have recruited patients with established disease fulfilling the old classification criteria. In our previous studies we have found that patients with shorter disease duration had a better clinical response to RTX.

Objectives: It is unknown whether treatment in very early SSc can affect long term outcomes. We aimed at assessing the effect of rituximab (RTX) in patients with very early SSc.

Methods: We report 2 cases where RTX was administered within 24 months from the appearance of Raynaud's.

Results: A 56 year-year-old female with an 18-month history of Raynaud's developed interstitial lung disease (ILD) with extensive ground glass lesions, a normal FVC (82\%) but decreased DLco (44\%). A diagnosis of SSc was made based on the presence of positive anti-centromere antibodies, puffy fingers, telangiectasias, positive capillaroscopy, ILD and Raynaud's. Six months following RTX treatment, her PFT's had increased (FVC 94\% and DLco 55\%) and chest HRCT showed an obvious improvement. One year later the patient no longer reported dyspnea. She remained on RTX for 5 years with no respiratory symptoms, stable PFTs and no evidence of disease progression. The second case is a 27-year old female with a 1-year history of Raynaud's, puffy fingers, positive anti-Scl70 and capillaroscopy. Within a few months her skin thickened rapidly reaching an MRSS of 12 and developed tendon friction rubs. Six months following RTX treatment skin thickening had almost resolved; she only had mild sclerodactyly. Tendon friction rubs disappeared. The patient received 8 consecutive RTX courses throughout a period of 4 years. During this period the patient remained in a steady clinical condition with no signs of disease progression.

Conclusion: It is generally accepted that autoimmunity/immune dysregulation and vasculopathy are the primary events in SSc that eventually trigger the fibrotic process. Based on this pathogenetic model, one can hypothesize that a therapeutic intervention very early in the disease course, prior to the appearance of fibrotic manifestations could potentially prevent permanent organ damage. Our data suggest that there may be a window of opportunity in SSc. This is why we propose that large scale, controlled studies assessing the efficacy of RTX (or other immune based therapies such as mycophenolate) in patients with very early disease are highly needed.

Disclosure of Interests: None declared

DOI: 10.1136/annrheumdis-2019-eular.3795

\section{AB0650 PERSPECTIVES AND UNMET NEEDS OF PATIENTS WITH POOR PROGNOSIS SYSTEMIC SCLEROSIS ON AUTOLOGOUS HEMATOPOIETIC STEM CELL TRANSPLANTATION CARE: A QUALITATIVE STUDY}

Carolijn de Bresser ${ }^{1}$, Julia Spierings ${ }^{1}$, Femke van Rhijn-Brouwer ${ }^{2}$

Arwen Pieterse $^{3}$, Madelon Vonk ${ }^{4}$, Alexandre Voskuyl ${ }^{5}$, Jeska de Vries-Bouwstra ${ }^{6}$, Marijke Kars ${ }^{7}$, Jacob M. van Laar'. ${ }^{1}$ University Medical Centre Utrecht, Department of Rheumatology and Clinical Immunology, Utrecht, Netherlands; ${ }^{2}$ University Medical Center Utrecht, Department of Rheumatology and Clinical Immunology and Department of Nephrology and Hypertension, Utrecht, Netherlands; ${ }^{3}$ Leiden University Medical Center (LUMC), Department of Medical Decision Making, Leiden, Netherlands; ${ }^{4}$ Radboud University Medical Center, Department of Rheumatology, Nijmegen, Netherlands; ${ }^{5}$ Amsterdam UMC, Amsterdam Rheumatology and Immunology Centre, Amsterdam, Netherlands; ${ }^{6}$ Leiden University Medical Center (LUMC), Department of Rheumatology, Leiden, Netherlands; ${ }^{7}$ Julius Center for Health Sciences and Primary Care, Department of Medical Humanities, Utrecht, Netherlands

Background: Autologous hematopoietic stem cell transplantation (HSCT) can induce long-term remission in patients with diffuse cutaneous systemic sclerosis (dcSSc). Nevertheless, it is an intensive treatment with risk of treatment-related mortality and associated adverse events requiring long-term hospital admission. Little is known about the psychosocial impact of HSCT on patients with dcSSc. Additionally, post-discharge care is currently not standardized.
Objectives: To gain more insight in the experiences of patients during and after HSCT and to identify unmet needs in current care.

Methods: Semi-structured interviews were conducted with dcSSc patients that underwent HSCT in four academic hospitals in the Netherlands. Interviews were transcribed verbatim and analyzed with NVIVO12.

Results: Eight patients were interviewed ( 5 males and 3 females, mean age 48 years (SD 10), mean disease duration 4.6 years (SD 1.9), mean time since HSCT 3.2 years (SD 2.4). At inclusion mean SHAQ was 1.3 (SD 1.1) and mean EQ5D-5L 0.86 (SD 0.21). While patients were satisfied with the frequent, low-threshold contacts with physicians and nurses, the decision-making process followed by HSCT and recovery was mentally taxing. Although patient education was experienced as extensive pretreatment, participants reported they only felt the real impact of it during therapy. Patients felt anxious throughout the hospital stay and they could not recall most events from that period. Feelings of losing control ove health and over time were frequently described. Support of family and friends was important during treatment. Apart from the practical aid, they provided emotional support, helping to cope with emotions and to find purpose. Furthermore, a specialized nurse involved in psychosocial support and care management was highly valued. Still, participants felt lonely and misunderstood due to invisible disabilities, such as fatigue and pain After HSCT, patients experienced more physical limitations than they expected, i.e. low energy levels and limited hand function. They worried about contracting infections and relapse risk. Professional rehabilitation was often hindered. There were unmet needs. Patients preferred to be better informed about what to expect after admission. Structured and written instructions should include themes such as self-management, practical tips and prognosis of recovery. Participants would advise peers to keep a diary and even take photos during HSCT and hospital stay, in order to help recollect and assimilate past events. Peer-support was recommended by most patients.

Conclusion: HSCT had a large impact, both physical and emotional. We identified unmet needs regarding patient education on rehabilitation and psychosocial support in the period following HSCT.

Disclosure of Interests: Carolijn de Bresser: None declared, Julia Spierings Grant/research support from: Boehringer Ingelheim, Femke van RhijnBrouwer: None declared, Arwen Pieterse: None declared, Madelon Vonk Grant/research support from: Madelon Vonk has received unrestricted research funds from Actelion and Therabel, Consultant for: Madelon Vonk was a consultant for Actelion, Boehringer-Ingelheim, Speakers bureau: Actelion, Boehringer-Ingelheim, Roche, Alexandre Voskuyl: None declared, Jeska de Vries-Bouwstra: None declared, Marijke Kars: None declared, Jacob M. van Laar Grant/research support from: Genentech, Consultant for: F. Hoffmann-La Roche

DOI: 10.1136/annrheumdis-2019-eular.2405

\section{AB0651 1 THE INFLUENCE OF RITUXIMAB THERAPY ON THE DYNAMICS OF THE BASIC PARAMETERS AND ACTIVITY OF SYSTEMIC SCLEROSIS}

Oxana Desinova, Mayya Starovoytova, Lidia P. Ananyeva, Liudmila Garzanova, Olga Koneva, Olga Ovsyannikova. V.A.Nasonova Institute of Rheumatology, Moscow, Russian Federation

Background: Systemic sclerosis (SSc) is an autoimmune disease characterized by skin and multi- organ involvements induced by overproduction of autoantibodiies by B cells.

Objectives: To assess the influence of Rituximab (RTX) therapy on the dynamics of the basic parameters and activity of SSc during long-term follow-up.

Methods: There were $90 \mathrm{SSc}$ patients (pts) in the prospective study. $75 \%$ were women. $57 \%$ pts had diffuse SSc. SSc was confirmed ACR EULAR criteria. The average duration of the disease was 5,8 years and of the study was 27 months. For the purpose of the analysis, a group of pts was selected that had no less than two evaluation points from 12 to 42 months and having received $2,9 \pm 1.1 \mathrm{~g} R T X$ in average during such period. The examination included both routine clinical laboratory parameters and special research. We evaluated the modified Rodnan skin score (mRSS), forced vital capacity (FVC), diffusing capacity of carbon monoxide (DLCO), the Valentini Disease Activity index, estimated glomerular filtration rate (eGFR) before and after completed RTX. All pts included in the study received treatment with vascular, anti-inflammatory and/or immunosuppressive medicines. The average dose of prednisolone was $11,8 \pm 4,4 \mathrm{mg} / \mathrm{day}$

Results: In general, more than $90 \%$ pts demonstrated different kinds of improvements. A reliable improvement was documented based on a number of indicators, which reflect typical features of the disease. Thus, skin induration was reduced (skin score decreased), lung functional capacity 
increased (increase of FVC of more than $5 \%$ ). The skin score was reliably decreased from $11,2 \pm 9,3$ to $6,2 \pm 4,9 \quad(p<0.0001)$, FVC was increased from $76,9 \pm 19,9$ to $84,7 \pm 20,9 \% \quad(p<0.0001)$, also the increase of DLCO from $46,3 \pm 18,3$ to $47,8 \pm 16,9 \%$ from the proper amount was detected. The Valentini Disease Activity index reliably reduced from $2,93 \pm 1,7$ to $1,38 \pm 1,2$ points $(p<0,0001)$. Under the RTX therapy, the decrease of glucocorticoids dose was insignificant, but statistically reliable. Thus, prednisolone dose reduction from $11,8 \pm 4,4$ to $9,22 \pm 3,2 \mathrm{mg} /$ day was achieved Clinically insignificant but reliable decline of eGFR from $100,2 \pm 23$ to 94,6 $\pm 22 \mathrm{ml} / \mathrm{min} / 1,73 \mathrm{~m} 2$ was detected.

Conclusion: Thus, RTX has a positive influence on the basic parameters of SSc, mainly on skin damage and lung functionality, reduces the general disease activity. The influence of the RTX on kidney function requires further long-term study.

Disclosure of Interests: None declared

DOI: 10.1136/annrheumdis-2019-eular.6700

\section{AB0652 QTC INTERVAL PROLONGATION IN A SCANDINAVIAN COHORT OF PATIENTS WITH IDIOPATHIC INFLAMMATORY MYOPATHIES AND SYSTEMIC SCLEROSIS: CORRELATIONS WITH CLINICAL VARIABLES}

Sine Søndergaard Korsholm ${ }^{1}$, Maryam Dastmalchi ${ }^{2}$, John Bonde Knudsen ${ }^{1}$, Axel Cosmus Diederichsen ${ }^{3}$, Ingrid E. Lundberg ${ }^{4}$, Daniel C. Andersson ${ }^{5}$, Nanna Witting ${ }^{6}$, Søren Jacobsen ${ }^{7}$, Tina Friis ${ }^{8}$, Markus E. Krogager ${ }^{6}$, Louise Pyndt Diederichsen ${ }^{1,7}$. ${ }^{1}$ Odense University Hospital, Department of Rheumatology, Odense, Denmark; ${ }^{2}$ Karolinska University Hospital, Department of Medicine, Rheumatology Unit, Stockholm, Sweden; ${ }^{3}$ Odense University Hospital, Department of Cardiology, Odense, Denmark; ${ }^{4}$ Karolinska Institutet, Department of Medicine, Rheumatology Unit, Stockholm, Sweden; ${ }^{5}$ Karolinska Institutet, Department of Physiology and Pharmacology, Stockholm, Sweden; ${ }^{6}$ Rigshospitalet, Department of Neurology, Copenhagen, Denmark; ${ }^{7}$ Rigshospitalet, Copenhagen University Hospital, Center for Rheumatology and Spine Diseases, Copenhagen, Denmark ${ }^{8}$ Statens Serum Institut, Department of Autoimmunology and Biomarkers, Copenhagen, Denmark

Background: Idiopathic inflammatory myopathies (IIM) are rare devastating diseases characterized by progressive muscle weakness and muscle fatigue. IIM frequently affects other organs pointing to IIM as a systemic inflammatory disease. Cardiac involvement is associated with poor prognosis. The symptoms are often subclinical and therefore overlooked. QTC prolongation has been detected in patients with systemic sclerosis (SSc). Autoantibodies are important diagnostic tools to confirm IIM and are present in approx. $60 \%$ of IIM patients. Autoantibodies are increasingly being recognized as markers for specific organ involvement. A biomarker for cardiac involvement has yet to be elucidated.

Objectives: The aim is to generate new knowledge about cardiac involvement in IIM detected by electrocardiography (ECG) and to evaluate possible associations between autoantibodies and cardiac involvement detected on ECG in a cohort of IIM patients compared with ECG changes in a cohort of SSc patients.

Methods: In a Scandinavian cohort, 263 IIM patients (130 polymyositis patients, 77 dermatomyositis patients, and 56 inclusion body myositis patients) and 102 SSc patients were investigated by ECG and basic cardiovascular and disease specific assessments according to international guidelines. IIM patients were tested for myositis specific autoantibodies (MSAs; anti-Jo-1, anti-PL-7, anti-PL-12, anti-OJ, anti-EJ, anti-SRP, anti-Mi2, anti-MDA5, anti-TIF1 $\gamma$, anti-NPX2, anti-SAE1, anti-HMGCR) and myositis associated autoantibodies (MAAs; anti-PM/Scl75, anti-PM/Scl100, antiRo52, anti-Ku, anti-cN1A)

Results: Twenty two IIM patients (8.49\%) had abnormal QRS duration versus one SSc patient $(1.06 \%)(P=0.012)$. SSc patients had significantly longer QTC duration than IIM patients (QTc $=432.7 \mathrm{~ms} \pm 22.2$ and $426.4 \mathrm{~ms} \pm 23.6$, respectively) $(P=0.03)$.

Multivariate regression analysis revealed that increased $\mathrm{C}$-reactive protein $(\mathrm{CRP})(\mathrm{P}=0.008)$, gender $(\mathrm{P}=0.002)$, and hypertension $(P=0.007)$ were associated with QTC duration in IIM patients. Likewise, pulmonary arterial hypertension was associated with QTC duration $(P<0.001)$ in SSc patients.

In analysis of pooled data for IIM patients and SSc patients, factors associated with QTc duration were increased CRP $(P=0.005)$, gender $(P=0.001)$, and hypertension $(P=0.01)$.

Conclusion: Both IIM patients and SSc patients had ECG changes though no particular pattern was shown. The findings support our hypothesis on cardiac involvement in IIM patients. No significant association was found between presences of either myositis specific or myositis associated autoantibodies and ECG changes. This could be due to the relatively low number of each autoantibody. There is a need to conduct larger prospective studies to identify a possible autoantibody for cardiac involvement in IIM.

Acknowledgement: The authors would like to thank all participating patients and the study personnel at the including centres.

Disclosure of Interests: Sine Søndergaard Korsholm: None declared, Maryam Dastmalchi: None declared, John Bonde Knudsen: None declared, Axel Cosmus Diederichsen: None declared, Ingrid E. Lundberg Grant/ research support from: Dr. Lundberg has received honoraria from Bristol Myers Squibb and Medlmmune and is currently receiving a research grant from Bristol Myers Squibb and from Astra Zeneca., Consultant for: She is a scientific advisor for Bristol Myers Squibb, and aTyr, Daniel C Andersson: None declared, Nanna Witting: None declared, Søren Jacobsen: None declared, Tina Friis Grant/research support from: Anti cN-1A ELISA kits and EUROLINE Autoimmune Inflammatory Myopathies 16 AG kits have been provided for a project free of charge from Euroimmun. Markus E. Krogager: None declared, Louise Pyndt Diederichsen: None declared

DOI: 10.1136/annrheumdis-2019-eular.2394

\section{AB0653 SERUM CARDIAC BIOMARKERS BUT NOT SUBCLINICAL CARDIOVASCULAR MAGNETIC RESONANCE ABNORMALITIES IN SYSTEMIC SCLEROSIS ASSOCIATE WITH THE DEVELOPMENT OF CARDIOVASCULAR EVENTS}

Raluca-Bianca Dumitru ${ }^{1}$, Lesley Anne Bissell ${ }^{1}$, Bara Erhayiem ${ }^{2}$, Graham Fent $^{2}$, Ananth Kidambi $^{2}$, Peter Swoboda ${ }^{2}$, Helena Donica ${ }^{3}$, Agata Burska ${ }^{1}$, John Biglands ${ }^{4}$, John Greenwood ${ }^{2}$, Francesco Del Galdo ${ }^{1}$, Sven Plein ${ }^{2}$ Maya Buch'. ' ${ }^{1}$ Leeds Institute of Rheumatic and Musculoskeletal Medicine, Leeds, United Kingdom; ${ }^{2}$ Leeds Institute of Cardiovascular and Metabolic Medicine, Leeds, United Kingdom; ${ }^{3}$ Department of Biochemical Diagnostics, Lublin, Poland; ${ }^{4}$ National Institute for Health Research, Leeds Biomedical Research Centre, Leeds, United Kingdom

Background: Primary cardiac disease in systemic sclerosis (SSc-PCD) is associated with a poor prognosis when clinically evident. Subclinical SScPCD is described in up to $2 / 3$ of SSc patients when sensitive methods are employed. The prognostic implication of these findings is not clear. Objectives: To describe the prevalence of cardiovascular magnetic resonance (CMR) abnormalities, their association with clinical phenotype and cardiac biomarkers, and whether cardiac biomarkers and CMR predict the development of cardiovascular (CV) events.

Methods: Patients fulfilling the ACR/EULAR criteria for SSc, with no CV disease (CVD), diabetes and no more than $1 \mathrm{CV}$ risk factor had 3Tesla CMR, including late gadolinium enhancement (LGE), myocardial perfusion, T1 mapping for T1 native and extracellular volume (ECV) quantification, and cardiac biomarkers measured. CV events were defined as episode of myocarditis, heart failure, rhythm disturbances and/or any echocardio graphic abnormalities including systolic dysfunction, diastolic dysfunction > grade 1 or regional wall motion abnormalities. 47 healthy volunteers (HV) with comparable age and gender served as controls.

Results: 83 SSc patients were recruited, median (IQR) age of 54 (49, 54 ), $84 \%$ females and $33 \%$ dcSSc, $40 \%$ had ILD, $24 \%$ a history of digital ulceration (HxDU) and $29 \%$ were Scl70+.

CMR showed higher ECV\% and T1 native, markers of diffuse fibrosis in SSc compared to HV [mean (SD) 30 (4) vs 25 (3), $\mathrm{p}=0.000 ; 1241$ (76) vs 1206 (55), $p=0.003$ ]. Lower myocardial perfusion reserve (MPR) was also noted in SSc patients [mean (SD) $2(0.8)$ vs 3 (1), $\mathrm{p}=0.000$ ]. None of the HV had LGE-fibrosis, whilst $16(20 \%)$ of the patients had a nonischemic LGE pattern. Left ventricular (LV) volume and function were similar between HV and SSc patients.

Presence of LGE associated with hsTnl and lower forced vital capacity (rho=0.283, $\mathrm{p}=0.032$; $\mathrm{rho}=-0.217, \mathrm{p}=0.5$ ). Multivariate analysis revealed an association of $T 1$ native with mRSS and CRP $(B=0.5, p=0.02 ; B=0.218$, $\mathrm{p}=0.049)$. ECV\% associated with NTproBNP and HxDU $(B=0.032$ $p=0.034 ; \quad B=0.310, p=0.005)$. $73 / 83$ had $\geq 1$ year available clinical and echocardiography follow up data with a mean (SD) duration of 24(20) months. $12 / 73$ had a CV event judged to be SSc-PCD. LV mass associated with $C V$ events $(p=0.043)$ (Table 1$)$, whilst univariate regression analysis showed that both hsTnl (OR=1.014, $\mathrm{p}=0.014)$ and NTproBNP $(\mathrm{OR}=1.005, p=0.026)$ but none of the CMR variables associated with the development of $\mathrm{CV}$ events.

Conclusion: Asymptomatic SSc patients with no overt CVD demonstrate both focal and diffuse fibrosis, which are associated with cardiac bio markers and markers of SSc disease activity. Whilst hsTnl and NTproBNP associated with the development of CV events, none of the measures of fibrosis did although a higher LV mass was noted in this 